\title{
An effective method of determining the residual stress gradients in a micro-cantilever
}

\begin{abstract}
A method of determining the micro-cantilever residual stress gradients by studying its deflection and curvature is presented. The stress gradients contribute to both axial load and bending moment, which, in prebuckling regime, cause the structural stiffness change and curving up/down, respectively. As the axial load corresponds to the even polynomial terms of stress gradients and bending moment corresponds to the the odd polynomial terms, the deflection itself is not enough to determine the axial load and bending moment. Curvature together with the deflection can uniquely determine these two parameters. Both linear analysis and nonlinear analysis of micro-cantilever deflection under axial load and bending moment are presented. Because of the stiffening effect due to the nonlinearity of (large) deformation, the difference between linear and nonlinear analyses enlarges as the micro-cantilever deflection increases. The model developed in this paper determines the resultant axial load and bending moment due to the stress gradients. Under proper assumptions, the stress gradients profile is obtained through the resultant axial load and bending moment.
\end{abstract}

\section{Introduction}

The successful fabrication and reliable operation of micro-structures strongly depend on the sufficiently rigorous understanding of their length-scale dependent and process-dependent mechanical properties (Srikar and Spearing 2003). For example, as a popular material for the fabrication of micro-structures, polysilicon is typically deposited by low-pressure chemical vapor

Y. Zhang · Y. Zhao $(\bowtie)$

State Key Laboratory of Nonlinear Mechanics (LNM), Institute of Mechanics, Chinese Academy of Sciences, Beijing, 100080 People's Republic of China

E-mail: yzhao@lnm.imech.ac.cn deposition (LPCVD). The vapor deposition causes either high compressive or tensile residual stress gradients, depending on the deposition process (Yang et al. 2000). The existence of residual stress (gradients) causes the change of equilibrium configuration and shifts of resonant frequencies of the micro-structures. Residual stress/strain becomes a parameter of fundamental importance in micro-structure, thin film, surface micromaching and improving reliability of micro-devices (Elbrecht et al. 1997; Fang and Wickert 1994, 1996). Tremendous effort has been infused to develop the measurement methods for the built-in residual stress/ strain in micro-structures. Devices like double-supported bridge/bridge array (Guckel et al. 1985; Elbrecht et al. 1997), bent-beam strain sensor (Gianchandani and Najafi 1996), micro-rotating-structure (Goosen et al. 1993), micro strain gauge (Lin et al. 1997), T- and Hshape structures (Allen et al. 1987; Mehregany et al. 1987), suspended microrings (Guckel et al. 1992), are developed for the measurement of the residual stress/ strain. Wafer curvature test is a popular technique for the evaluation of residual stress in non-integrated, constrained structures like film deposited on the substrate (Srikar and Spearing 2003; Elbrecht et al. 1997), which has the advantage of evaluating (film) structure residual stress state without knowing the mechanical properties of the (film) structure. The sensitivity of beam resonant frequency to the residual strain is used to measure the shifts of the resonant frequency to determine the residual strain (Ikehara et al. 2001). The onset of the buckling of micro-structures with known geometries is used to directly measure the residual strain level (Elbrecht et al. 1997; Guckel et al. 1985). Because of the buckling sensitivity to structural imperfection and experimental difficulties of telling buckled and unbuckled microstructures, Fang and Wickert (1994) use micro-structure postbuckling equilibrium configuration to determine the residual stress. Physically, the cause of residual stress can be explained by revised Thomas-Fermi-Dirac (TFD) model as the result of the difference of the electron density at the boundary of the atoms (EDBA) 
(Cheng and Cheng 1998). Qian et al. extend the EDBA model to determine the residual stress in microelectromechanical systems (MEMS) multi-layer structure (Qian et al. 2002).

Although the techniques for the residual stress/strain measurement mentioned above have the fortes such as direct and in-situ measurement, the limits and experimental difficulties are also obvious. For example, for wafer curvature test technique, it is mainly confined to non-integrated, constrained structures. For the buckling technique, telling the onset of buckling or the buckled, unbuckled structures is very difficult (Elbrecht et al. 1997; Fang and Wickert 1994), the micro strain gauge design is suggested to change the gauge dimensions to avoid buckling/postbuckling measurement scenario (Lin et al. 1997). Buckling and postbuckling equilibrium configuration are very sensitive to the initial imperfection of the micro-structures. For postbuckling analysis, the initial imperfection must be known a priori. Measuring the small imperfection of micro-structure can be very difficult and such initial geometric condition can be a significant error source (Srikar and Spearing 2003). In the postbuckling analysis of Fang and Wickert (1994), the imperfection shape is assumed. Bent-beam strain sensor measures the beam tip displacement to determine the residual stress. However, for bent-beam strain sensor, the exerted axial load is related to the bending moment via the specific structural configuration (Gianchandani and Najafi 1996; Que et al. 2001). As we will see later in this paper, the axial load and bending moment generally are two independent parameters. Measuring the tip displacement alone is not enough to determine the micro-structure residual stress state for general application. For EDBA model application, EBDA at interface must be known to determine the residual stress gradients (Qian et al. 2002).

The deflection profile of a micro-structure can be obtained through non-contact interferometric profilometry system using the fringe pattern generated by interference. And the fringe can be digitalized by CCD camera using Wyko-3D system for further analysis. Once the deflection profile of a micro-structure is measured, the slope and curvature can be easily calculated. By studying the deflection and curvature, the model presented in this paper can uniquely determine the resultant axial load and bending moment due to residual stress gradients.

\section{Model development}

Figure 1 shows scanning electron microscope (SEM) image of the deflection of a micro polysilicon cantilever under residual stress gradients influence. Generally speaking, the complicated state of residual stress depends on the fabrication process and the residual stress varies through the thickness (Lin and PugaczMuraszkiewicz 1972; Kiesewetter et al. 1992). For

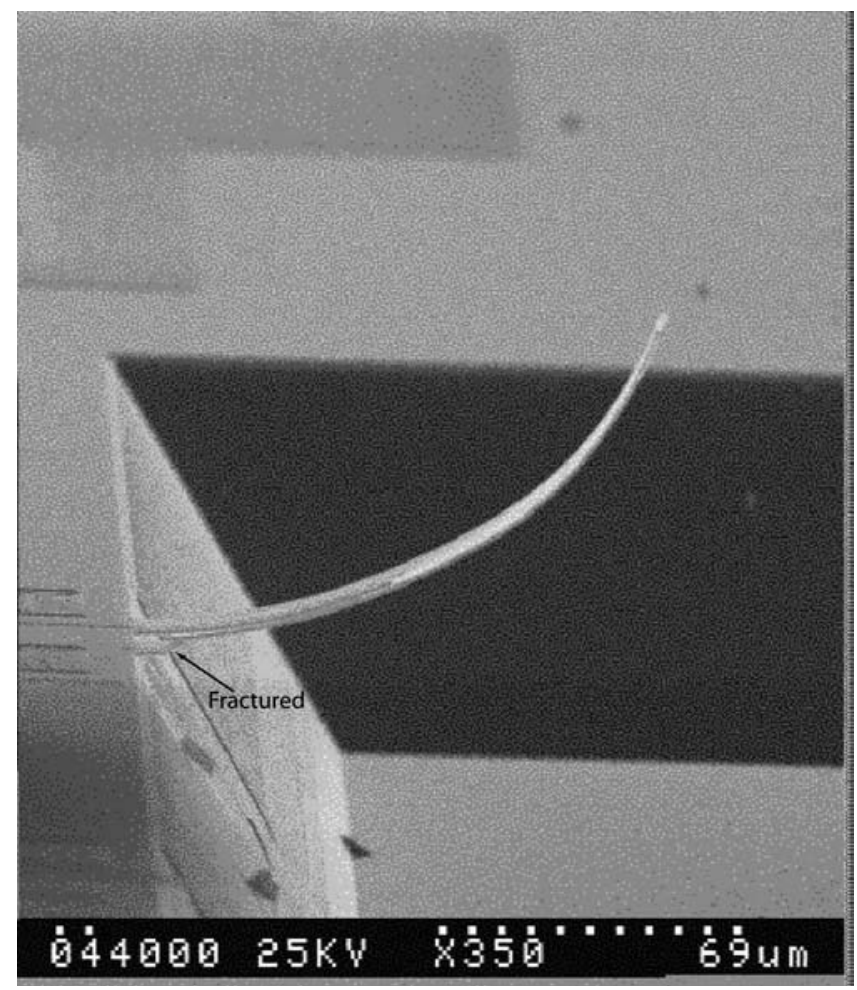

Fig. 1 SEM image of the cantilevered microbeam deflection under stress gradients. There are two beams, the front one is broken due to the large stress gradients

quantitative analysis, the residual stress field is assumed to have the following polynomial distribution (Fang and Wickert 1996; Yang et al. 1995)

$\sigma=\sum_{k=0}^{N} \sigma_{k}\left(\frac{y}{h / 2}\right)^{k}$

where $y$ is the coordinate across the thickness and $y \in$ $y \in\left(\frac{-h}{2}, \frac{h}{2}\right) . h$ is the thickness and neutral plane is chosen at $y=0 . \sigma_{0}$ is the constant mean stress and $\sigma_{k}(k=1,2$, $3, \ldots, N)$ is gradient stress. As the thickness becomes smaller, the stress gradient becomes particularly acute (Fang and Wickert 1996). For this paper, we analyze the cantilever beam deflection, the beam dimensions and coordinate system are shown in Fig. 2a. For rectangular beam, the axial load $T_{o}$ due to the residual stress gradients is as follows

$$
\begin{aligned}
T_{o} & =\int_{A} \sigma \mathrm{d} A \\
& =b \int_{-h / 2}^{h / 2} \sum_{k=0}^{N} \sigma_{k}\left(\frac{y}{h / 2}\right)^{k} \mathrm{~d} y=\frac{b h}{2} \sum_{k=0}^{N} \frac{\sigma_{k}}{k+1}\left[1^{k}+(-1)^{k}\right]
\end{aligned}
$$

and the bending moment $m$ due to the residual stress is 

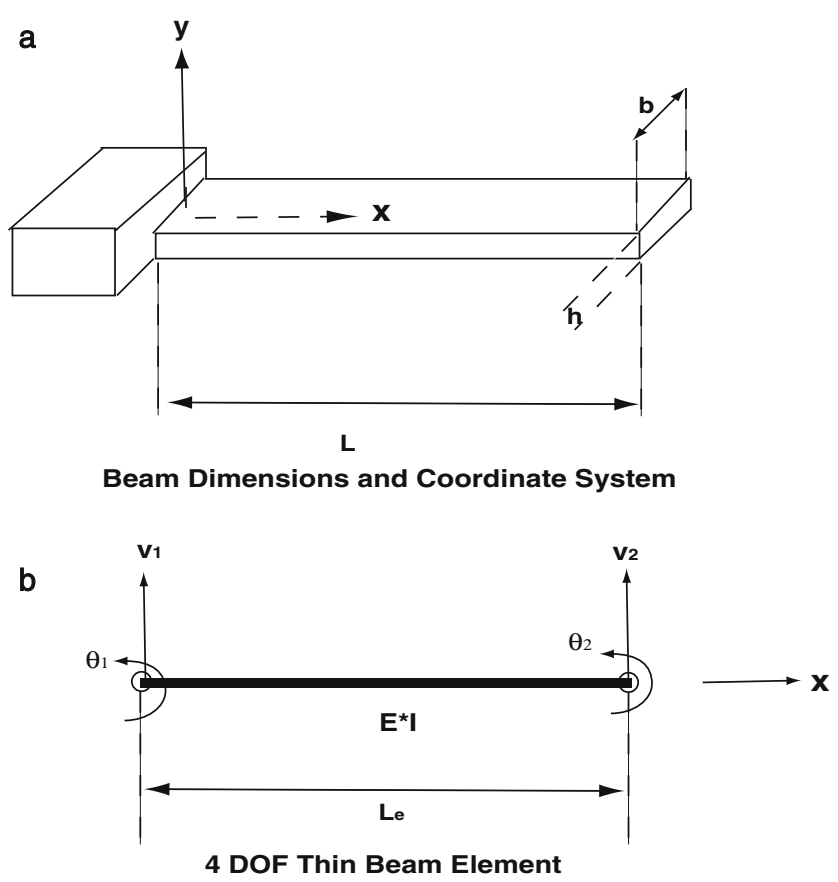

Fig. 2 Schematic diagrams of the cantilever beam dimensions, coordinates and a 4 DOF thin beam element

$$
\begin{aligned}
m & =\int_{A} y \sigma \mathrm{d} A \\
& =b \int_{-h / 2}^{h / 2} y \sum_{k=0}^{N} \sigma_{k}\left(\frac{y}{h / 2}\right)^{k} \mathrm{~d} y \\
& =\frac{b h^{2}}{4} \sum_{k=0}^{N} \frac{\sigma_{k}}{k+2}\left[1^{k}-(-1)^{k}\right],
\end{aligned}
$$

where $b$ is the width of the beam. Equations 2 and 3 show that $T_{o}$ is associated only with even polynomial terms of $\sigma$ and $m$ only with odd polynomial terms. Therefore, $T_{o}$ and $m$ are two parameters due to the residual stress and they are independent to each other. In a sense, the effects of the residual stress in Eq. 1 are converted and included into two parameters $T_{o}$ and $m$, which are the actual parameters used in the governing equation. The beam potential energy of bending is

$U_{b}=\frac{1}{2 E^{*} I} \int_{0}^{L} M^{2} \mathrm{~d} x$

where $E^{*}$ is effective modulus. For 2-D thin structure like film under biaxial stress state, $E^{*}$ is biaxial modulus (Klein 2000; Freund et al. 1999), $E^{*}=E /(1-v) \quad(E$ Young's modulus and $v$ Poisson's ratio). For wide beam (plate under cylindrical bending), $E^{*}=E /\left(1-v^{2}\right)$ (Timoshenko and Woinowsky-Krieger 1959; Abdel-Rahman et al. 2002). For narrow beam, $E^{*}$ is simply taken as $E$. $L$ is the beam length and $I\left(I=b h^{3} / 12\right)$ is the moment inertia of the cross section of rectangular beam. For beam, when $\left(I / A L^{2}\right)<0.001$ ( $A$ is cross section area and $A=b h$ for rectangular beam), the following linear moment-curvature relation is sufficiently accurate even for relatively large deflection (Thurman and Mote 1969)

$M=m+E^{*} I v_{x x}$,

where $v$ is the beam deflection displacement. For the potential energy of stretching, we divide it into two parts. $U_{\mathrm{s} 1}$, the part due to the axial load $T_{o}$, is

$U_{\mathrm{s} 1}=\frac{T_{o}}{2} \int_{0}^{L} v_{x}^{2} \mathrm{~d} x$

The other part, $U_{\mathrm{s} 2}$, which accounts for the nonlinearity of the large deflection (McDonald and Raleigh 1955), is as follows

$U_{s 2}=\frac{A E^{*}}{2 L}\left[\frac{1}{2} \int_{0}^{L} v_{x}^{2} \mathrm{~d} x\right]^{2}$.

By applying principle of minimum potential energy $\left[\delta\left(U_{b}+U_{\mathrm{s} 1}+U_{\mathrm{s} 2}\right)=0\right]$, the following equation is obtained

$$
\begin{aligned}
& \int_{0}^{L}\left(m \delta v_{x x}+E^{*} I v_{x x} \delta v_{x x}+T_{o} v_{x} \delta v_{x}\right) \mathrm{d} x+\frac{A E^{*}}{2 L} \int_{0}^{L} v_{x}^{2} \mathrm{~d} x \times \int_{0}^{L} v_{x} \delta v_{x} \mathrm{~d} x \\
& =0
\end{aligned}
$$

For this nonlinear equation, finite element analysis (FEA) method is applied to discretize the system and Newton-Rhapson method is used to numerically obtain the solution. The element is a 4 degree of freedom (DOF) thin beam element shown in Fig. $2 b$.

For linear analysis, the stretching energy of $U_{\mathrm{s} 2}$ part is omitted. By applying principle of minimum potential energy $\left(\delta\left(U_{b}+U_{\mathrm{s} 1}\right)=0\right)$ and after two more integration by parts procedures, the equation of equilibrium is obtained as follows

$E^{*} I v_{x x x x}-T_{o} v_{x x}=0$.

The four boundary conditions for the cantilever are also obtained

$v(0)=0, \quad v_{x}(0)=0, \quad E^{*} I v_{x x}(L)+m=0$,
$E^{*} I v_{x x x}(L)-T_{o} v_{x}(L)=0$.

For $T_{o}>0$ case (tensile load), Eq. 9 has such solution form

$v=A \cosh (\alpha x)+B \sinh (\alpha x)+C x+D$

for $T_{o}<0$ case (compressive load)

$v=A \cos (\alpha x)+B \sin (\alpha x)+C x+D$

and $T_{o}=0$ case 
$v=A x^{3}+B x^{2}+C x+D$

$\alpha$ is defined as $\alpha=\sqrt{\left|\frac{T_{o}}{E^{*} \mid}\right|} . A, B, C$ and $D$ are the constants determined by the boundary conditions for each case.

\section{Results and discussions}

All the results are obtained by setting $L=50 \mu \mathrm{m}$, $h=2 \mu \mathrm{m}$ and $E^{*}=66 \mathrm{GPa}$. From the expressions of $T_{o}$, $m$ and potential energies, it is not hard to conclude that the parameter $b$, the beam width, does not have any influence on the beam deflection (in Eqs. 8, 9 and 10, $b$ is canceled out). For statement and computation convenience, we keep $b$ in our derivation and set it as $1 \mathrm{~m}$. Figure 3 shows the convergence study of FEA method on nonlinear analysis. The deflections computed by 5 , 10, 15 elements are shown together in Fig. 3. All other results of nonlinear analysis in this paper are obtained by using ten elements, which offers sufficiently accurate results. Figure 4 shows the deflection difference between linear analysis and nonlinear analysis under different loading scenarios. $T_{o}$ is fixed as zero and $m$ is taken as $-2.93 \times 10^{-4}$ and $-4.4 \times 10^{-4} \mathrm{~N} \mathrm{~m}$, respectively. As the deflection increases, the difference enlarges. The nonlinear analysis results are always smaller than those of linear analysis due to the nonlinear stiffness hardening effect (Nayfeh and Mook 1979).

The purpose of this paper is to use the beam tip displacement and curvature to determine the residual stress state inside the beam. While, the computations (Eqs. 8 and 9) can only be carried out when $T_{o}$ and $m$ are known.
The idea is that we use either Eq. 8 or Eq. 9 to compute many beam deflection cases with different $T_{o}$ and $m$ combinations, then compare those curves to experimentally measured one. If the computed curve matches the measured one, the corresponding $T_{o}(\mathrm{~s})$ and $m(\mathrm{~s})$ are found. And we give an example to illustrate this procedure. Figure 5 shows the beam tip displacement $v(L)$ computed by Eqs. 11, 12 and 13 as $T_{o}$ changes from $0.2 P_{\text {cri }}$ to $-2.8 P_{\text {cri }}$ with the step size of $-3 P_{\text {cri }} / 40$ and $m$ changes from $-6 \times 10^{-4}$ to $6 \times 10^{-4} \mathrm{~N}$ m with the step size of $3 \times 10^{-5} \mathrm{~N} \mathrm{~m}$. There are $41 \times 41$ meshes. $P_{\text {cri }}=-\left(\left(\pi^{2}\right.\right.$ $\left.\left.E^{*} I\right) /\left(4 L^{2}\right)\right)$ is the buckling load (compressive) for cantilever beam with uniform cross section. If a measured curve with tip displacement of $5.03 \mu \mathrm{m}$ (an arbitrarily chosen number), from Fig. 5, there are four cases with the tip displacements within $1 \%$ difference from the given tip displacement of $5.03 \mu \mathrm{m}$. The four cases are c1: $v(L)=5.071 \mu \mathrm{m}\left(T_{o}=66.225 \mathrm{~N}, m=-4.675 \times\right.$ $\left.10^{-4} \mathrm{~N} \mathrm{~m}\right), \mathrm{c} 2: v(L)=5.053 \mu \mathrm{m}\left(T_{o}=72.739 \mathrm{~N}, m=\right.$ $\left.-4.95 \times 10^{-4} \mathrm{~N} \mathrm{~m}\right), \mathrm{c} 3: v(L)=5.036 \mu \mathrm{m}\left(T_{o}=79.253 \mathrm{~N}, m\right.$ $\left.=-5.225 \times 10^{-4} \mathrm{~N} \mathrm{~m}\right)$ and $\mathrm{c} 4: v(L)=5.019 \mu \mathrm{m}\left(T_{o}=\right.$ $\left.85.766 \mathrm{~N}, m=-5.5 \times 10^{-4} \mathrm{~N} \mathrm{~m}\right)$. The value $(1 \%)$ of difference tolerance can be changed to account for the factors such as measurement error, gravity (Yang et al. 1995), which makes measured value deviating from real one. It should be pointed out that the four cases which are picked according to the criterion of their tip displacement with specific difference tolerance from the given value are also dependent on the mesh size. For different mesh size, the case number can be different. Just by the tip displacement criterion, only four cases from $41 \times 41$ cases are picked. From c1 to c4, axial load $T_{o}$ (tensile) increases, which stiffens the structure, as the result, $m$ correspondingly increases to achieve the approximately same
Fig. 3 The convergence study of FEA for nonlinear analysis as the element number increases

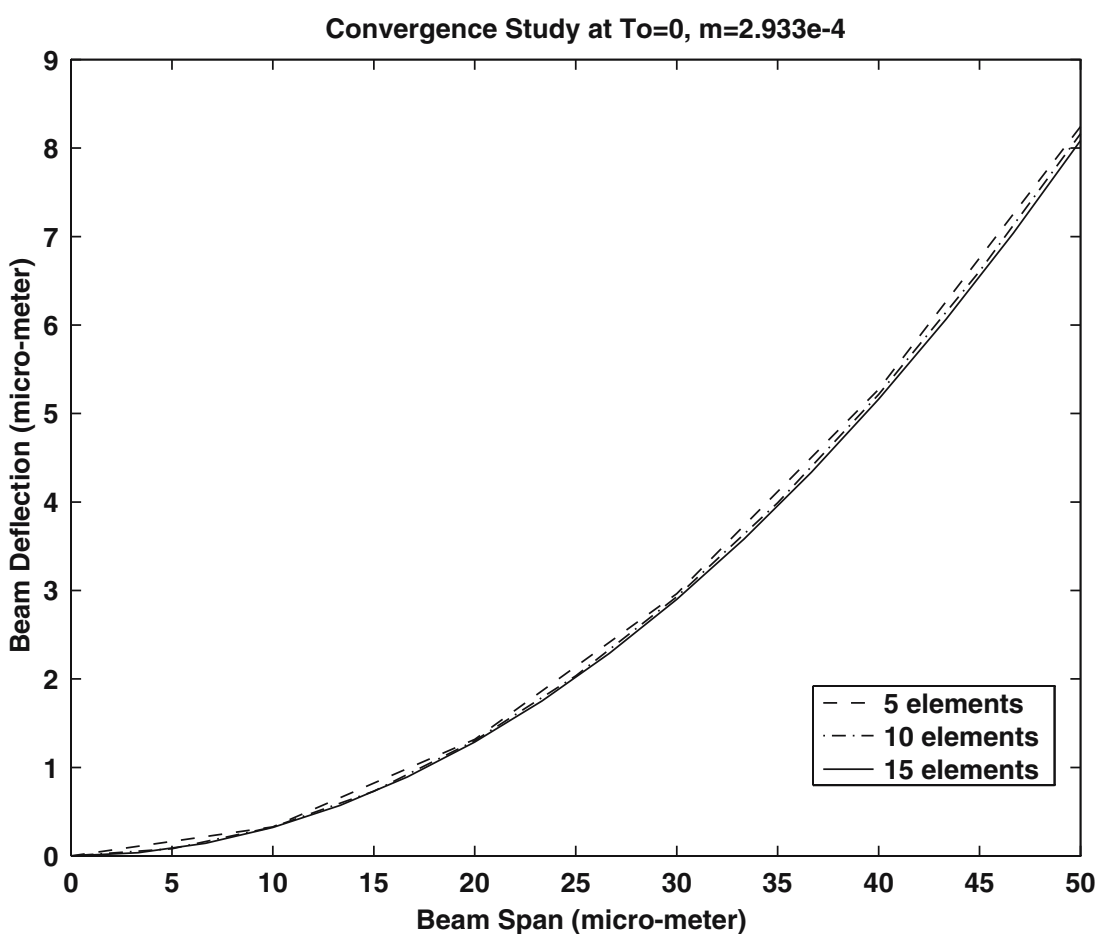


Fig. 4 The comparison of linear analysis and nonlinear analysis on the beam deflection under the different two sets of $T_{o}$ and $m$
Fig. 5 The beam tip displacement of linear analysis as $T_{o}$ changes from $0.2 P_{\text {cri }}$ to $2.8 P_{\text {cri }}$ and $m$ changes from $-6 \times 10^{-4}$ to $6 \times 10^{-4} \mathrm{~N} \mathrm{~m}$
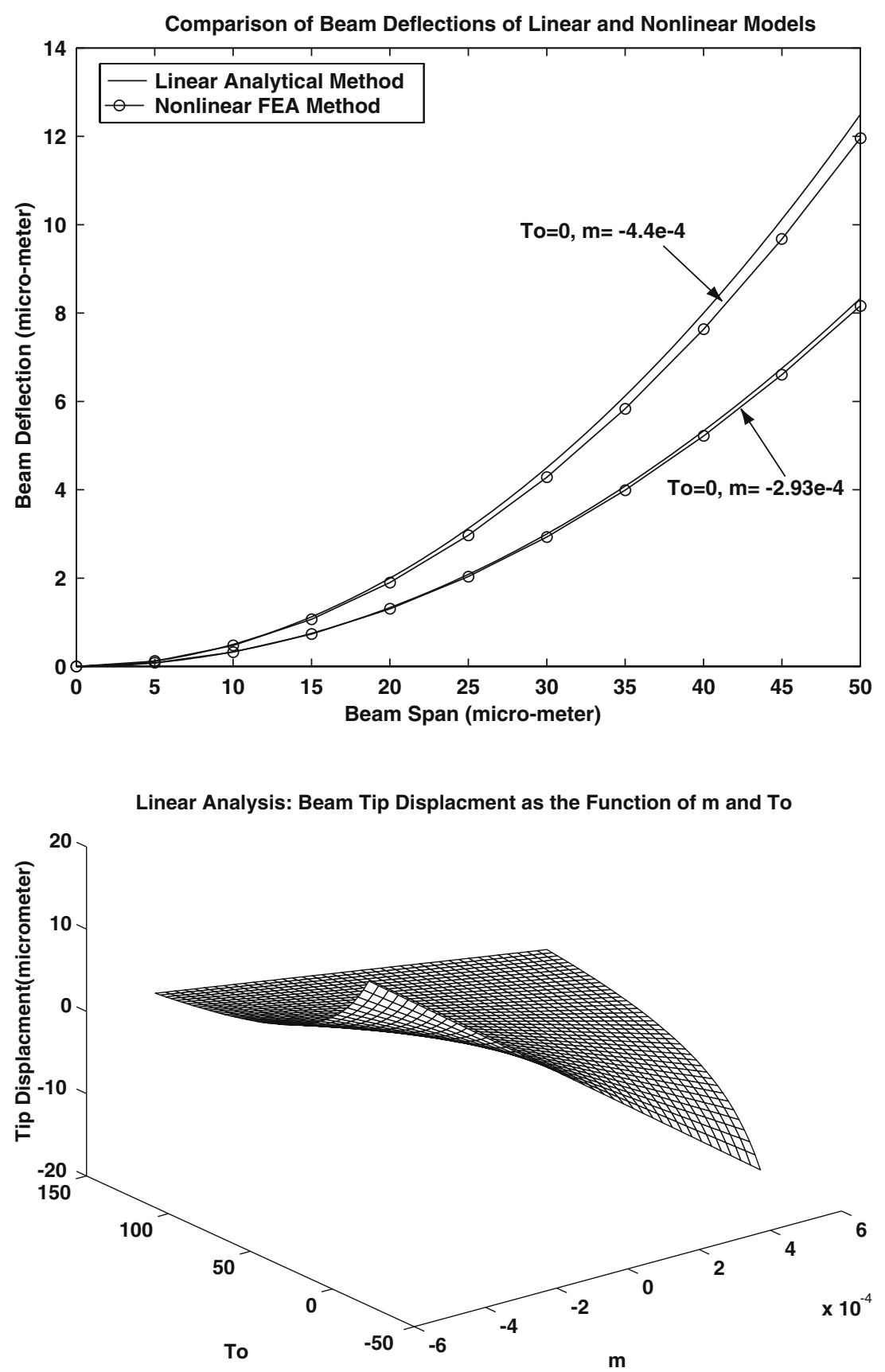

tip displacement. Figure 6 shows the deflections of these four cases. As the beam deflection depends on both $T_{o}$ and $m$, which generally are two independent parameters, the tip displacement criterion alone cannot determine the residual stress state. Even for the whole deflection curve along the beam span, it is very hard to tell the deflection curve difference for the four cases in Fig. 6. As we notice that for these four cases, the bending moment $m$ is well separated and $M$ $\left(M=m+E^{*} I v_{x x}\right)$ is associated the curvature of the deflection curve. So for the four deflection curves with very similar deflection shapes but different $m \mathrm{~s}$, it is well possible that their curvatures can be very different (at least for some parts of the beam span). The curvature here is approximated as $v_{x x}$. For linear analysis, the curvature can be analytically obtained through Eqs. 11, 12 and 13 according to $T_{o}$ value. For nonlinear analysis, the curvature is numerically obtained. Figure 7 shows the curvatures of the four cases deflection curves. Compared with the deflection curves in Fig. 6, the curvature curves, especially at the beam free-end, are much better separated. So if given the measured curvature at the free-end, $T_{o}$ and $m$ are uniquely determined together with the measured tip displacement. As it is shown in Fig. 4, the difference of linear analysis and nonlinear analysis enlarges as the deflection increases. Thus the linear analysis becomes less accurate for larger deflection case. To compare this difference, we define $f$ as $f=\left(v_{\mathrm{nl}} / v_{\mathrm{ln}}\right) \cdot v_{\mathrm{nl}}$ is the beam tip displacement by 
Fig. 6 The comparison of the four beam deflection cases with the approximately same tip displacement but under different $T_{o}$ and $m$
Fig. 7 The comparison of the curvatures for the four beam deflection cases with the same tip displacement but under different $T_{o}$ and $m$
The Four Cases Beam Deflection Comparison
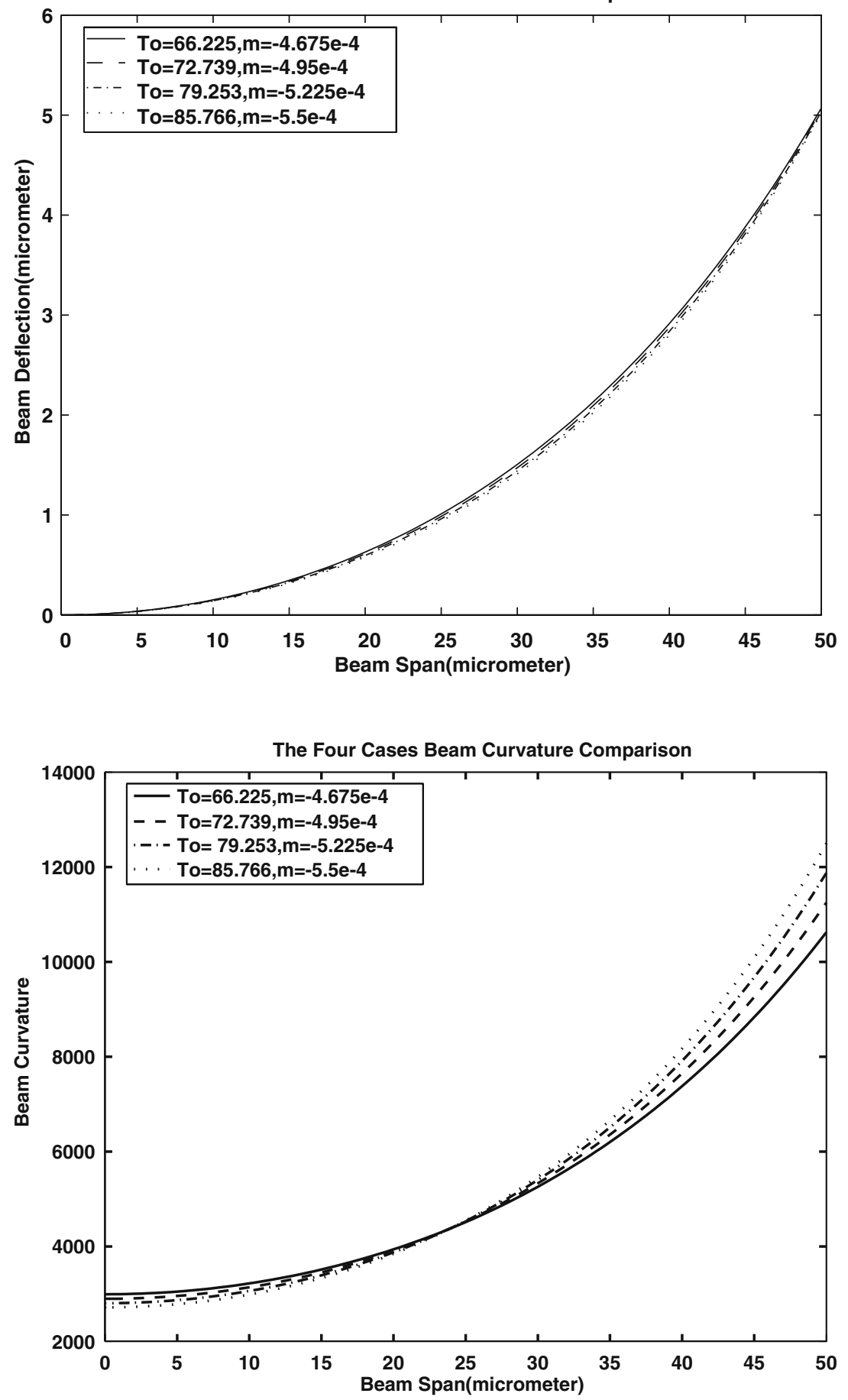

nonlinear analysis and $v_{\mathrm{ln}}$ is that by linear analysis. Figure 8 shows $f$ versus $T_{o}$ and $m$. $T_{o}, m$ ranges and mesh sizes in Fig. 8 are the same as those in Fig. 5. As $T_{o}$ range is away from buckling load (the beam is in prebuckling regime), the beam deflection is zero for both linear analysis and nonlinear analysis when $m=0$. For this $0 / 0$ case, $f$ is set as 1 in Fig. 8 . It is not surprised that all $f$ values are less than or equal to 1 because of aforementioned nonlinear stiffening effect. Because compressive load softens the structure, at $T_{o}=0.2 P_{\text {cri }}$, $m=-6 \times 10^{-4} \mathrm{~N} \mathrm{~m}$ and $T_{o}=0.2 P_{\text {cri, }}, m=6 \times 10^{-4} \mathrm{~N} \mathrm{~m}$, the beam has the largest deflections. Therefore, $f$ has the smallest value at these two points, which indicates the difference of linear and nonlinear analysis is the greatest at the two points.

All of analysis above actually determines only the resultant axial load $T_{o}$ and bending moment $m$ due to the stress gradients $\left(\sigma_{k} \mathrm{~s}\right) . \sigma_{k} \mathrm{~s}$ are related to $T_{o}$ and $m$ through Eqs. 2 and 3. Therefore, only two $\sigma_{k}$ s can be determined for one measurement (of tip displacement 
Fig. 8 The comparison of the beam tip displacement computed by nonlinear analysis and linear analysis as $T_{o}$ changes from $0.2 P_{c r i}$ to -2.8 $P_{c r i}$ and $m$ changes from $-6 \times 10^{-4}$ to $6 \times 10^{-4} \mathrm{~N} \mathrm{~m}$. $f$ of the $z$-axis is the ratio of tip displacement computed by nonlinear analysis and tip displacement computed by linear analysis
Comparison of Nonlinear Analysis and Linear Analysis on Beam Tip Displacment

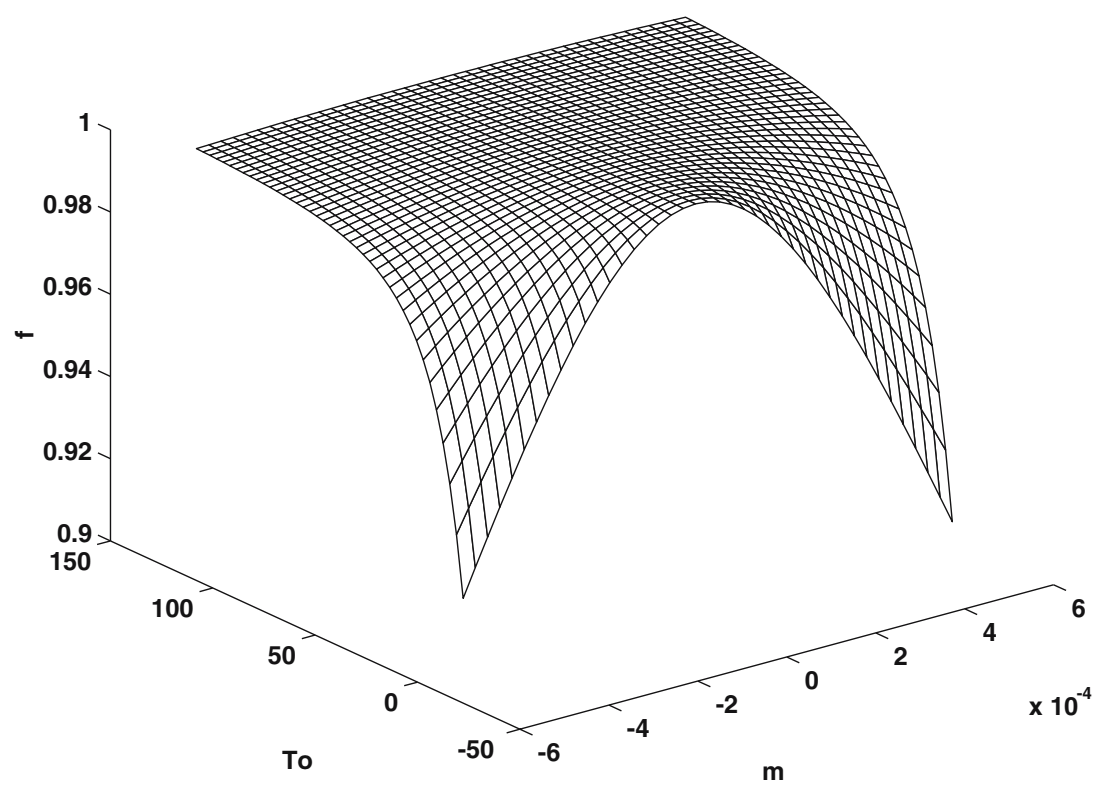

and curvature) of a given structural dimensions. For approximation, Fang and Wickert (1996) use $\sigma_{0}$ to represents all symmetric (even) polynomial terms and $\sigma_{1}$ for all anti-symmetric (odd) polynomial terms. If we assume $\sigma_{k}$ s are fixed and change the beam dimension, for example, changing the beam thickness, new $T_{o}$ and $m$ will be obtained by following the exact procedure we describe above. Two more linear equations of $\sigma_{k}$ s will be obtained again through Eqs. 2 and 3. Therefore, two more $\sigma_{k}$ s can be determined. More and more $\sigma_{k} \mathrm{~s}$ can be determined by changing the beam dimension(s) for more measurements. This is the approach of determining stress gradients used by Yang et al. (1995).

In our analysis, $T_{o}$ is chosen to be far away from buckling load on purpose though Eq. 8 is suitable for postbuckling analysis. When $m=0$, Eq. 8 corresponds to prebuckling/buckling/postbuckling analysis for the beam without imperfection case and $m \neq 0$ corresponds to the beam with imperfection case (there is some mathematical difference between Eq. 8 and the equation of Fang and Wickert 1996). In postbuckling regime, there are three equilibria(one unstable, the other two stable) and axial load alone can cause the deflection of the structure with no imperfection $(m=0$ case). This causes the great difficulty of using the method we propose to determine the micro-structure residual stress state in postbuckling regime. While, because such postbuckling analysis work has been done by Fang and Wickert (1996), our nonlinear analysis here aims more to offer modification and adjustment for linear analysis and evaluate the applicability range of linear analysis in prebuckling regime. Our nonlinear analysis does not consider the coupling effect of longitudinal and transverse displacements, so it is restricted to the case of very small slenderness ratio (Nayfeh and Mook 1979). For the comprehensive reading of slenderness ratio influence on the kinematic assumptions of nonlinear analysis, the reader should refer to Nayfeh and Mook's book (Nayfeh and Mook 1979). For very large stress gradients case, as shown in Fig. 1, the front beam is fractured under large stress gradients action. Before fracture, plastic deformation happens and our analysis is elastic analysis. Although our model does not take those factors like gravity, pressure, beam spatial dimensional variation, stress gradients longitudinal variation, which affect beam deflection, into account, those factors are not that hard to be incorporated into the model. However, the boundary conditions listed by Srikar and Spearing as no. 1 error source in MEMS testing methods (Srikar and Spearing 2003), may be the major error source for the application of our method. For "cantilevered" beam, both FEA computation and experimental measurement by Fang and Wickert (1996) show that there is a rotating angle depending on stress gradients at the "fixed" end. This factor will be extremely difficult to be incorporated into our model if not impossible.

\section{Conclusion}

The resultant axial load and bending moment due to the stress gradients are proposed to be determined by measuring the micro-cantilever tip displacement and curvature. The micro-cantilever deflection profile can be measured via non-contact inferometric systems. Unlike other techniques like measuring resonant frequency shift, buckling/postbuckling, etc, the possible damage, extra devices like excitation, loading instruments, spectrum analyzer etc, and the error source due to the ease of these instrumentation and fixturing (Srikar and Spearing 2003) can be avoided by our method. The limits of our 
model are also discussed. Because of the uncertainty due to the boundary conditions, micro-fabricated structure dimensions, fabrication defects, micro-cracks, etc, it is of great importance and necessity to use micro-devices for direct and in situ measurement.

Acknowledgements The supports from the Distinguished Young Scholar Fund of National Natural Science Foundation of China (NSFC, Grant No. 10225209), the key project from Chinese Academy of Sciences (Grant No. KJCX-SW-L2) and the NSFRGC joint project (Grant No. 50131160739) are greatly appreciated.

\section{References}

Abdel-Rahman E, Younis MI, Nayfeh AH (2002) Characterization of the mechanical behavior of an electrically actuated microbeam. J Micromech Microeng 12:759-766

Allen MG, Mehregany M, Howe RT, Senturia SD (1987) Microfabricated structures for the in situ measurement of residual stress, Young's modulus, and ultimate strain of thin films. Appl Phys Lett 51:241-243

Cheng KJ, Cheng SY (1998) Analysis and computation of the internal stress in thin film. Prog Nat Sci 8:679-689

Elbrecht L, Storm U, Catanescu R, Binder J (1997) Comparison of stress measurement techniques in surface micromaching. J Micromech Microeng 7:151-154

Fang W, Wickert JA (1994) Post buckling of micromaching beams. J Micromech Microeng 4:116-122

Fang W, Wickert JA (1996) Determining mean and gradient residual stresses in thin films using micromachined cantilevers. J Micromech Microeng 6:301-309

Freund LB, Floro JA, Chason E (1999) Extensions of the Stoney formula for substrate curvature to configurations with thin substrate or large deformations. Appl Phys Lett 74:1987-1989

Gianchandani Y, Najafi K (1996) Bent-beam strain sensors. J Microelectromech Syst 5:52-58

Goosen JFL, van Drieënhuizen BP, French PJ, Wolffenbuttel RF (1993) Stress measurement structures for for micromachined sensors. In: Tech. Dig. 7th int. conf. on Solid State Sensors and Actuators, Transducer '93 (Yokohama, Japan), June 7-10, pp 783-786

Guckel H, Randazzo T, Burns DW (1985) A simple technique for the determination of mechanical strain in thin films with application to polysilicon. J Appl Phys 57:1671-1675
Guckel H, Burns O, Rutigliano, Lowell E, Choi B (1992) Diagnostic microstructures for the measurements of intrinsic strain in thin films. J Micromech Microeng 2:86-95

Ikehara T, Zwijze RAF, Ikeda K (2001) New method for an accurate determination of residual strain in polycrystalline silicon films by analysing resonant frequencies of micromachined beams. J Micromech Microeng 11:55-60

Kiesewetter L, Zhang JM, Houdeau D, Stecknborn A (1992) Determination of Young's moduli of micromechanical thin films using the resonance method. Sensor Actuat A 35:153-159

Klein CA (2000) How accurate are Stoney's equation and recent modifications. J App Phys 88:5487-5489

Lin SCH, Pugacz-Muraszkiewicz I (1972) Local stress measurement in thin thermal $\mathrm{SiO}_{2}$ films on $\mathrm{Si}$ substrates. J App Phys 43:119-125

Lin L, Pisano AP, Howe RT (1997) A micro strain gauge with mechanical amplifier. J Microelectromech Syst 6:313-321

McDonald PH, Raleigh NC (1955) Nonlinear dynamic coupling in a beam vibration. J Appl Mech 22:573-578

Mehregany M, Howe RT, Senturia SD (1987) Novel microstructures for the in situ measurement of mechanical properties of thin films. J Appl Phys 62:3579-3584

Nayfeh AH, Mook DT (1979) Nonlinear oscillations. Wiley, New York

Qian J, Zhao YP, Zhu RZ, Yu TX (2002) Analysis of residual stress gradient in MEMS multi-layer structure. Int $\mathbf{J}$ Nonlin Sci Numer Simulat 3:727-730

Que L, Park JS, Gianchandani Y (2001) Bent-beam electrothermal actuators-part I: single beam and cascaded devices. J Microelectromech Syst 5:52-58

Srikar VT, Spearing SM (2003) A critical review of microscale mechanical testing methods used in the design of microelectromechanical systems. Exp Mech 43:238-247

Thurman AL, Mote CD (1969) Free, periodic, nonlinear oscillation of an axially moving strip. J Appl Mech 36:83-89

Timoshenko S, Woinowsky-Krieger S (1959) Theory of plates and shells, 2nd edn. McGraw-Hill Book Company Inc, New York

Yang EH, Yang SS, Yoo SH (1995) A technique for quantitative determination of the profile of the residual stress along the depth of $p^{+}$silicon films. Appl Phys Lett 67:912-914

Yang J, Kahn H, He A, Philips SM, Heuer A (2000) A new technique for producing large-area as-deposited zero-stress LPCVD polysilicon films: the multipoly process. J Microelectromech Syst 9:485-494 